\title{
Heavy hadron spectroscopy: a quark model perspective
}

\author{
J. Vijande ${ }^{\mathrm{a}}$, A. Valcarce ${ }^{\mathrm{b}}$, T.F. Caramés ${ }^{\mathrm{b}}$, H. Garcilazo ${ }^{\mathrm{c}}$ \\ ${ }^{a}$ Departamento de Física Atómica, Molecular y Nuclear, Universidad de Valencia (UV) and IFIC (UV-CSIC), Valencia, Spain. \\ ${ }^{b}$ Departamento de Física Fundamental, Universidad de Salamanca, 37008 Salamanca, Spain \\ ${ }^{c}$ Escuela Superior de Física y Matemáticas, Instituto Politécnico Nacional, Edificio 9, 07738 México D.F., Mexico
}

\begin{abstract}
We present recent results of hadron spectroscopy and hadron-hadron interaction from the perspective of constituent quark models. We pay special attention to the role played by higher order Fock space components in the hadron spectra and the connection of this extension with the hadron-hadron interaction. The main goal of our description is to obtain a coherent understanding of the lowenergy hadron phenomenology without enforcing any particular model, to constrain its characteristics and learn about low-energy realization of the theory.
\end{abstract}

(C) 2012 Published by Elsevier Ltd.

Keywords: Heavy hadrons, Constituent quark model, Many-quark systems

\section{Introduction}

For almost thirty years after the discovery of the $J / \psi$ and its excitations, the so-called November revolution [1], heavy hadron spectroscopy was at rest. Paraphrasing Lord Kelvin famous speech [2], by 2003 there were only two clouds on the horizon obscuring the beauty and clearness of the dynamical theory. In the hadron spectra these two clouds were on the one hand the missing resonance problem, i.e., all quark models predict a proliferation of excited states which have not been measured, and, on the other hand, the observation by BaBar of an open-charm meson, the $D_{s J}^{*}(2317)$, whose properties were quite different from those predicted by quark potential models.

Since those peaceful days an increase on both amount and quality of experimental data has shown quite a different picture, far more involved and convoluted, with the observation of the well-known $X(3872)$, the $X(3940), Y(3940)$, $Z$ (3930), $Y(4140)$, and several other states. Based on Gell-Mann conjecture [3] the hadronic experimental data were classified either as $q \bar{q}$ or $q q q$ states according to $S U(3)$ irreducible representations, nowadays this hypothesis may be in question. Therefore, the study of the role played by higher order Fock space components in the hadron spectra, allowed by the Gell-Mann classification, is an interesting issue to address.

In this talk we give an overview of a project for getting a coherent understanding of the low-energy hadron phenomenology from the perspective of constituent quark models [4]. We will make emphasis on three different aspects. Firstly, we will address the study of meson spectroscopy in an enlarged Hilbert space considering many-quark components. This seems nowadays unavoidable to understand the experimental data and it builds a bridge towards the description in terms of hadronic degrees of freedom. Secondly, the same scheme used to describe the hadron spectroscopy should be valid for describing the low-energy meson-meson scattering. In other words, contributions to the hadron spectroscopy arising from many-quark components allowed in the Gell-Mann scheme, should also be recovered by means of hadron-hadron scattering using a full set of states. Finally, we will review our recent efforts to describe the structure of heavy baryons containing charm or bottom quarks. 


\section{Meson spectroscopy beyond the naive quark-model}

Many-quark systems present a richer, and therefore more complicated, color structure than standard hadrons built with a quark-antiquark pair or three quarks. While the color wave function for ordinary mesons and baryons leads to a single vector, in the case of four-quark states there are different vectors driving to a singlet color state out of either colorless or colored quark-antiquark two-body components. Thus, when dealing with four-quark states a basic important question is whether we are in front of a single colorless meson-meson molecule or a compact state, defined as a system with two-body colored components. Whereas the first structure would be natural in the naive quark model, the second one would open a new area in hadron spectroscopy.

Let us start by defining a physical channel as a four-quark state made of two quark-antiquark color singlets. In Ref. [5] the formalism to evaluate the probability of the different physical channels for an arbitrary four-quark wave function was derived. For this purpose any hidden-color vector of a four-quark color basis, i.e., vectors with nonsinglet internal color couplings, was expanded in terms of singlet-singlet color vectors. Such a procedure gives rise to a wave function expanded in terms of nonorthogonal vectors belonging to different orthogonal basis, and therefore the determination of the probability of physical channels becomes cumbersome. Such nonorthogonal expansions are common in other fields of science, like chemical physics [6], however they have not been properly discussed until now in the context of the multiquark phenomenology, i.e., color chemistry. We have derived the two hermitian operators that are well-defined projectors on the physical singlet-singlet color states $|11\rangle_{c}$ and $\left|1^{\prime} 1^{\prime}\right\rangle_{c}$. Using these operators the probabilities for finding singlet-singlet color components for an arbitrary four-quark state $|\Psi\rangle$ are given by,

$$
\begin{aligned}
P^{|\Psi\rangle}([11]) & =\frac{1}{2\left(1-\left.\left.\right|_{c}\left\langle 11 \mid 1^{\prime} 1^{\prime}\right\rangle_{c}\right|^{2}\right)}[\langle\Psi|P \hat{Q}| \Psi\rangle+\langle\Psi|\hat{Q} P| \Psi\rangle] \\
P^{|\Psi\rangle}\left(\left[1^{\prime} 1^{\prime}\right]\right) & =\frac{1}{2\left(1-\left.\left.\right|_{c}\left\langle 11 \mid 1^{\prime} 1^{\prime}\right\rangle_{c}\right|^{2}\right)}[\langle\Psi|\hat{P} Q| \Psi\rangle+\langle\Psi|Q \hat{P}| \Psi\rangle],
\end{aligned}
$$

where $P(\hat{P})$ and $Q(\hat{Q})$ are the projectors over the different color vectors, $|11\rangle_{c}\left(\left|1^{\prime} 1^{\prime}\right\rangle_{c}\right)$ and $|88\rangle_{c}\left(\left|8^{\prime} 8^{\prime}\right\rangle_{c}\right)$ respectively.

The stability of a four-quark state relies on $\Delta_{E}=E_{4 q}-E\left(M_{1}, M_{2}\right)$, the energy difference between its mass and that of the lowest two-meson threshold. It is important to emphasize the relevance of comparing four-quark energies with respect to thresholds obtained using the same quark-quark interaction as in the four-quark case. Only in doing so one may guarantee that no spurious bound states are obtained. $\Delta_{E}<0$ indicates that all fall-apart decay channels are forbidden, and therefore one has a proper bound state. $\Delta_{E} \geq 0$ will indicate that the four-quark solution corresponds to an unbound threshold (two free mesons). As we will discuss below, between these two limits one may find molecular states.

The four-body $Q Q \bar{n} \bar{n}$ ( $Q$ stands for a heavy $c$ or $b$ quark and $n$ for a light $u, d$ or $s$ quark) Schrödinger equation has been solved using two independent methods, the hyperspherical harmonic formalism [7] and a variational approach

\begin{tabular}{|c|ccccc|}
\hline$(S, I, L=0)$ & $(0,1)$ & $(1,1)$ & $(1,0)$ & $(1,0)$ & $(0,0)$ \\
Flavor & $c c \bar{n} \bar{n}$ & $c c \bar{n} \bar{n}$ & $c c \bar{n} \bar{n}$ & $b b \bar{n} \bar{n}$ & $b b \bar{n} \bar{n}$ \\
\hline Energy (MeV) & 3877 & 3952 & 3861 & 10395 & 10948 \\
Threshold & $\left.D D\right|_{S}$ & $\left.D D^{*}\right|_{S}$ & $\left.D D^{*}\right|_{S}$ & $\left.B B^{*}\right|_{S}$ & $\left.B_{1} B\right|_{P}$ \\
$\Delta_{E}(\mathrm{MeV})$ & +5 & +15 & -76 & -217 & -153 \\
\hline$\left.P[\overline{3} 3\rangle_{c}^{12}\right]$ & 0.333 & 0.333 & 0.881 & 0.974 & 0.981 \\
$P\left[|6 \overline{6}\rangle_{c}^{12}\right]$ & 0.667 & 0.667 & 0.119 & 0.026 & 0.019 \\
\hline$P\left[|11\rangle_{c}\right]$ & 0.556 & 0.556 & 0.374 & 0.342 & 0.340 \\
$P\left[|88\rangle_{c}\right]$ & 0.444 & 0.444 & 0.626 & 0.658 & 0.660 \\
\hline$P_{M M}$ & 1.000 & - & - & - & 0.254 \\
$P_{M M^{*}}$ & - & 1.000 & 0.505 & 0.531 & - \\
$P_{M^{*} M^{*}}$ & 0.000 & 0.000 & 0.495 & 0.469 & 0.746 \\
\hline
\end{tabular}

Table 1. Four-quark state properties for selected quantum numbers. The notation $M_{1} M_{2} \mid \ell$ stands for mesons $M_{1}$ and $M_{2}$ with relative orbital angular momentum $\ell \cdot P\left[|\overline{3} 3\rangle_{c}^{12}\left(|6 \overline{6}\rangle_{c}^{12}\right)\right]$ and $P\left[|11\rangle_{c}\left(|88\rangle_{c}\right)\right]$ stand for the probability of the color components in different basis $[8]$. 

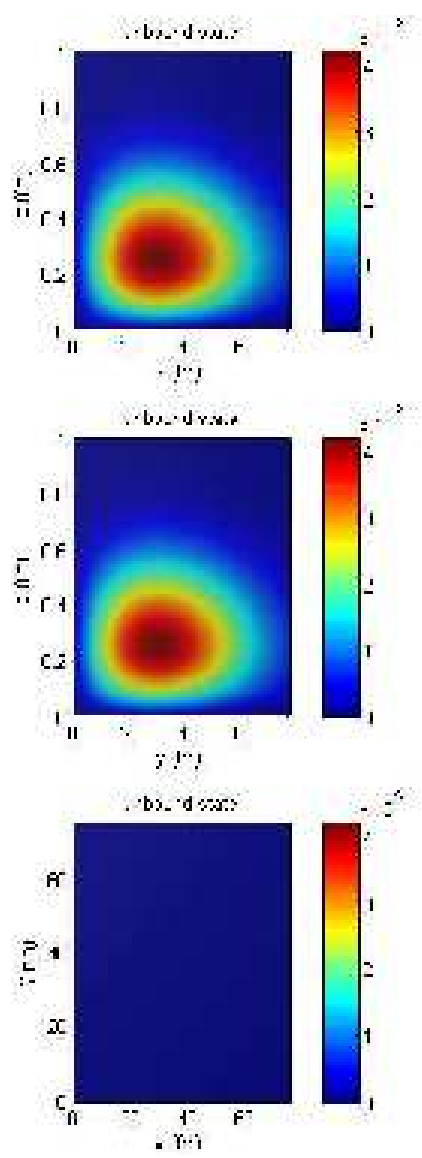
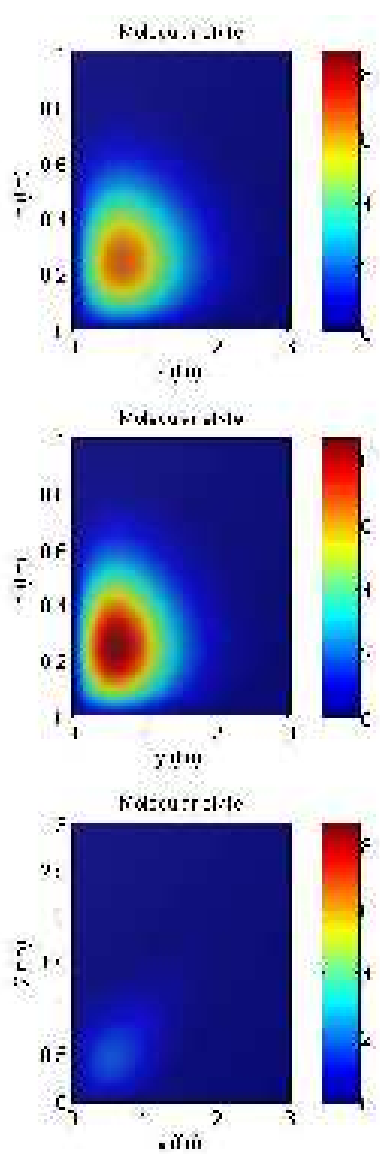
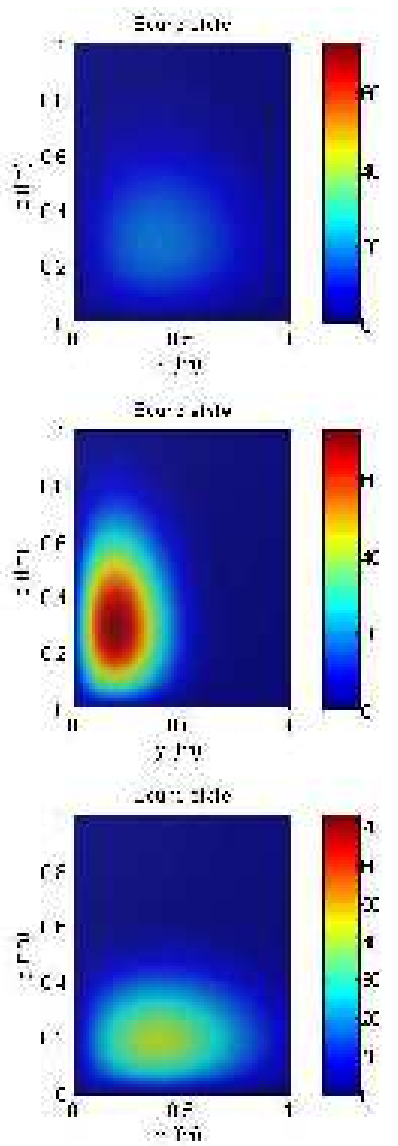

Figure 1. Position probability distributions corresponding to a unbound: $(S=1, I=1) \operatorname{cc} \bar{n} \bar{n}$, a molecular: $(S=0, I=1) c c \bar{n} \bar{n}$, and a bound state: $(S=1, I=0) b b \bar{n} \bar{n}$.

based on generalized gaussians [8]. All possible quantum numbers for both double charm and double bottom fourquark states were evaluated. Among all possible combinations, in the charm sector only one bound state with quantum numbers $(I) J^{P}=(0) 1^{+}$has been found. We show in Table 1 a summary of the results obtained for several bound and unbound four-quark states in the bottom and charm sectors. One can see how, independently of the binding energy, all of them contain a sizable octet-octet color component. Let us first of all concentrate on the two unbound states. Using the formalism of Ref. [5] one can evaluate the probability of the different physical channels: $P_{M M}$, two pseudoscalar mesons, $P_{M^{*} M^{*}}$, two vector mesons, and $P_{M M^{*}}$, a pseudoscalar and a vector meson. One can see how four-quark unbound states are represented by two isolated mesons. Let us now turn to the bound states. In contrast to the results obtained for unbound states, the probabilities in several of the allowed physical channels are relevant. Thus, it becomes clear how the bound state must be generated by an interaction that is not present in the asymptotic channel, sequestering probability from a single singlet-singlet color vector due to the interaction between color octets. Such systems are clear examples of compact four-quark states, in other words, they cannot be expressed in terms of a single physical channel.

To illustrate in more detail the differences observed in the calculated four-quark wave functions we depict in Fig. 1 the position probability distributions defined as

$$
R\left(r_{\alpha}, r_{\beta}\right)=r_{\alpha} r_{\beta} \sum_{i} \int_{V}\left|R_{i}\left(\vec{r}_{\alpha}, \vec{r}_{\beta}, \vec{r}_{\gamma}\right)\right|^{2} d \vec{r}_{\gamma} d \Omega_{r_{\alpha}} d \Omega_{r_{\beta}},
$$

where $R_{i}\left(\vec{r}_{\alpha}, \vec{r}_{\beta}, \vec{r}_{\gamma}\right)$ are the four-quark radial wave functions. We present results for a unbound, a molecular and a 
bound state, showing the position distribution for the different planes $\left(r_{\alpha}, r_{\beta}\right)=(z, x),(z, y)$, and $(x, y)$. Clear differences among them can be observed. The position distribution for the unbound case spreads in the $x$ and $y$ Jacobi coordinates up to $60 \mathrm{fm}$, while the bound and molecular systems are restricted to the region below $3 \mathrm{fm}$ (molecular) and $1 \mathrm{fm}$ (bound). In the $(x, y)$ plane the unbound state is so widely spread that the values for the position distribution are three orders of magnitude lower than in the $(z, y)$ and $(z, x)$ cases, and therefore they will not appear in the picture unless artificially magnified. In the case of the molecular state a long range tail propagating in the $x=y$ region can be observed contrary to the constrained values obtained for bound systems.

In a recent investigation, the $Q \bar{Q} n \bar{n}$ Schrödinger equation has been solved accurately using the hyperspherical harmonic formalism [9]. All possible quantum numbers were discussed by means of different constituent quark models widely used in the literature. No compact bound states were found for any set of quantum numbers nor any constituent quark model. Thus, independently of the quark-quark interaction and the quantum numbers considered, the system evolves to a well separated two-meson state. In any manner one can claim for the existence of a compact bound state for the $c \bar{c} n \bar{n}$ system.

Unfortunately, close to a threshold, methods based on a series expansion fail to converge since arbitrary large number of terms are required to determine the wave function. For this reason, we have solved the two-body LippmannSchwinger equation for negative energies using the Fredholm determinant method, looking for attractive channels that may contain a meson-meson molecule, that permitted us to obtain robust predictions even for zero-energy bound states [10]. To do so we have started from a physical system made of two mesons, $M_{1}$ and $M_{2}$, with quantum numbers $(I) J^{P C}$ in a relative $S$-wave. Then, in general, there is a coupling to the $M_{1} M_{2} D$-wave and to any other two-meson system that can couple to the same quantum numbers $(I) J^{P C}$. Thus, the Lippmann-Schwinger equation for the $M_{1} M_{2}$ scattering becomes

$$
t_{\alpha \beta ; J I}^{\ell_{\alpha} s_{\alpha}, \ell_{\beta} s_{\beta}}\left(p_{\alpha}, p_{\beta} ; E\right)=V_{\alpha \beta ; J I}^{\ell_{\alpha} s_{\alpha}, \ell_{\beta} s_{\beta}}\left(p_{\alpha}, p_{\beta}\right)+\sum_{\gamma} \sum_{\ell_{\gamma}=0,2} \int_{0}^{\infty} p_{\gamma}^{2} d p_{\gamma} V_{\alpha \gamma ; J I}^{\ell_{\alpha} s_{\alpha}, \ell_{\gamma} s_{\gamma}}\left(p_{\alpha}, p_{\gamma}\right) G_{\gamma}\left(E ; p_{\gamma}\right) t_{\gamma \beta ; J I}^{\ell_{\gamma} s_{\gamma}, \ell_{\beta} s_{\beta}}\left(p_{\gamma}, p_{\beta} ; E\right),
$$

where $\alpha \equiv \beta \equiv M_{1} M_{2}, \gamma$ stands for any intermediate two-body state that can couple to the (I) $J^{P C}$ quantum numbers, $t$ is the two-body scattering amplitude, $J, I$, and $E$ are the angular momentum, isospin and energy of the two-body system, $\ell_{\alpha} s_{\alpha}, \ell_{\gamma} s_{\gamma}$, and $\ell_{\beta} s_{\beta}$ are the initial, intermediate, and final orbital angular momentum and spin, respectively, and $p_{\gamma}$ is the relative momentum of the two-body system $\gamma$. To solve the scattering problem we have derived a mesonmeson potential in the Born-Oppenheimer approach from the basic $\bar{q} q$ interaction used to study the four-quark system with the hyperspherical harmonic formalism or the generalized gaussian variational approach.

We have analyzed all positive-parity channels made by $S$-wave interacting mesons up to $J^{P}=2^{+}$in the $Q Q \bar{n} \bar{n}$ sector. Only the channel $(I) J^{P}=(0) 1^{+}$is attractive enough to be bound [11]. This is the same state that was found to be bound in the four-quark study. As can be seen in Fig. 2 the $D D^{*}$ and $D^{*} D^{*}$ potentials are attractive, however none of them is bound by itself. A bound state is obtained when one considers the coupling between the $D D^{*}$ and the $D^{*} D^{*}$ systems and solves the coupled-channel problem accordingly. This mixing at short distances will reconstruct the octet-octet color vector component of a particular basis as shown in Table 1.

When moving to the bottom sector one finds four more candidates for observation. They are, ordered by their binding energy: $(I) J^{P}=(0) 1^{+},(0) 0^{+},(1) 3^{-}$and $(0) 1^{-}$. The interest in these states is increasing as for the first time there are chances to observe such large mass exotic states in quite a near future. LHC may discover tetraquark states via gluon-gluon fusion due to both large number of events and their unique signature in the detectors [12]. RHIC might identify hadronic molecular states by means of relativistic heavy ion collisions by employing the coalescence model for hadron production [13]. If any of these suggestions gets real, the new data related to these double charm or bottom exotic systems will have a huge impact on heavy quark spectroscopy.

In the $Q \bar{Q} n \bar{n}$ case, $D \bar{D}$ and charmonium-light two-mesons systems get coupled. A few channels are slightly attractive (Fredholm determinant smaller than one), $D \bar{D}$ with $(I) J^{P C}=(0) 0^{++}, D \bar{D}^{*}$ with $(0) 1^{++}$and $D^{*} \bar{D}^{*}$ with $(0) 0^{++},(0) 2^{++}$, and $(1) 2^{++}$. The remaining ones are either repulsive or have zero probability to contain a bound state or a resonance. The only bound state appears in the $(I) J^{P C}=(0) 1^{++}$channel as a consequence of the coupling between $D \bar{D}^{*}$ and $J / \Psi \omega$ two-meson channels. It has a binding energy in the range $0-1 \mathrm{MeV}$, and therefore in agreement with the experimental measurements of the $X(3872)$. The corresponding isovector channel becomes repulsive due to the coupling to the lightest channel that includes a pion. Therefore, the existence of charged partners for the $X(3872)$ can be discarded as well as a partner in the bottom sector [14]. 


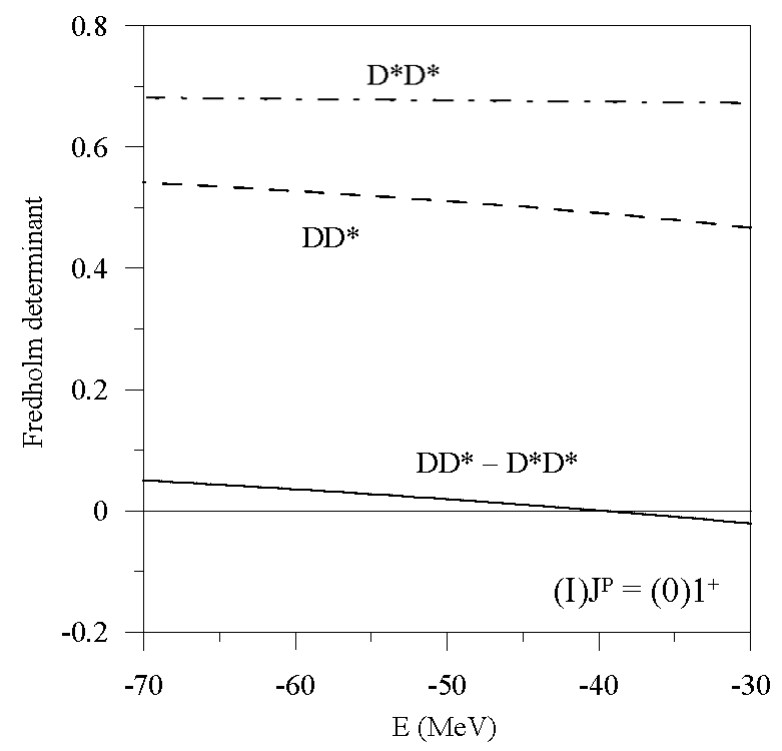

Figure 2. Fredholm determinant for the $(I) J^{P}=(0) 1^{+} c c \bar{n} \bar{n}$ system. The dashed line corresponds to the calculation considering only $D D^{*}$ singletsinglet color states. The dashed-doted line stands for the results considering only the $D^{*} D^{*}$ system. The solid line represents the results including the coupling $D D^{*} \leftrightarrow D^{*} D^{*}$.

Although the last analysis has been performed by means of a particular quark interacting potential, the conclusions derived are general. They mainly rely on using the same hamiltonian to describe tensors of different order, two- and four-quark components in the present case. When dealing with a complete basis, any four-quark deeply bound state has to be compact. Only slightly bound systems could be considered as molecular. Unbound states correspond to a two-meson system. A similar situation would be found in the two baryon system, the deuteron could be considered as a molecular-like state with a small percentage of its wave function on the $\Delta \Delta$ channel, whereas the $H$-dibaryon would be a compact six-quark state. When working with central forces, the only way of getting a bound system is to have a strong interaction between the constituents that are far apart in the asymptotic limit (quarks or antiquarks in the present case). In this case the short-range interaction will capture part of the probability of a two-meson threshold to form a bound state. This can be reinterpreted as an infinite sum over physical states. This is why the analysis performed here is so important before any conclusion can be made concerning the existence of compact four-quark states beyond simple molecular structures.

Lets conclude this section by emphasizing that at short distances identical quarks can recouple to different vectors of the Hilbert space. If any of them is not considered, a fundamental ingredient of the calculation would be neglected. Such effect would never happen when dealing with hadronic degrees of freedom. Therefore, the need to incorporate the complete Hilbert space is evident in both approaches. If the model parameters were fitted to some observables they would necessarily include the effect of a restricted Hilbert space and one should never use them for different quantum numbers without arriving to wrong conclusions, loosing thus any predictive power. We can therefore conclude that formalisms based on four-quark and meson-meson configurations are fully compatible whenever all the relevant basis vectors are taken into account.

\section{Heavy baryon spectroscopy}

Heavy baryons containing a single heavy quark are particularly interesting. The light degrees of freedom (quarks and gluons) circle around the nearly static heavy quark. Such a system behaves as the QCD analogue of the familiar hydrogen bounded by the electromagnetic interaction. When the heavy quark mass $m_{Q} \rightarrow \infty$, the angular momentum of the light degrees of freedom is a good quantum number. Thus, heavy quark baryons belong to either SU(3) 


\begin{tabular}{|cc||ccccc|ccccc|}
\hline \multirow{3}{*}{ State } & $J^{P}$ & {$[15]$} & Exp. & {$[17]$} & {$[18]$} & {$[19]$} & {$[15]$} & Exp. & {$[17]$} & {$[18]$} & {$[19]$} \\
\hline$\Lambda_{i}$ & $1 / 2^{+}$ & 2285 & 2286 & 2285 & 2268 & 2297 & 5624 & 5619 & 5638 & 5612 & 5622 \\
& $1 / 2^{+}$ & 2785 & 2765 & 2865 & 2791 & 2772 & 6106 & & 6188 & 6107 & 6086 \\
& $1 / 2^{-}$ & 2627 & 2595 & 2635 & 2625 & 2598 & 5947 & 5912 & 5978 & 5939 & 5930 \\
& $3 / 2^{+}$ & 3061 & & 2930 & 2887 & 2874 & 6388 & & 6248 & 6181 & 6189 \\
\hline$\Sigma_{i}$ & $1 / 2^{+}$ & 2435 & 2454 & 2455 & 2455 & 2439 & 5807 & 5811 & 5845 & 5833 & 5805 \\
& $1 / 2^{+}$ & 2904 & & 3025 & 2958 & 2864 & 6247 & & 6370 & 6294 & 6202 \\
& $1 / 2^{-}$ & 2772 & 2765 & 2805 & 2748 & 2795 & 6103 & & 6155 & 6099 & 6108 \\
& $3 / 2^{+}$ & 2502 & 2518 & 2535 & 2519 & 2518 & 5829 & 5833 & 5875 & 5858 & 5834 \\
\hline \multirow{2}{*}{$\Xi_{i}$} & $1 / 2^{+}$ & 2471 & 2471 & 2467 & 2492 & 2481 & 5801 & 5793 & 5806 & 5844 & 5812 \\
& $1 / 2^{\prime+}$ & 2574 & 2578 & 2567 & 2592 & 2578 & 5939 & & 5941 & 5958 & 5937 \\
& $1 / 2^{-}$ & 2799 & 2792 & 2792 & 2763 & 2801 & 6109 & & 6116 & 6108 & 6119 \\
& $3 / 2^{+}$ & 2642 & 2646 & 2647 & 2650 & 2654 & 5961 & 5945 & 5971 & 5982 & 5963 \\
\hline$\Omega_{i}$ & $1 / 2^{+}$ & 2699 & 2698 & 2675 & 2718 & 2698 & 6056 & 6054 & 6034 & 6081 & 6065 \\
& $1 / 2^{+}$ & 3159 & & 3195 & 3152 & 3065 & 6479 & & 6504 & 6472 & 6440 \\
& $1 / 2^{-}$ & 3035 & & 3005 & 2977 & 3020 & 6340 & & 6319 & 6301 & 6352 \\
& $3 / 2^{+}$ & 2767 & 2768 & 2750 & 2776 & 2768 & 6079 & & 6069 & 6102 & 6088 \\
\hline
\end{tabular}

Table 2. Masses, in MeV, of charmed and bottom baryons.

antisymmetric $\overline{\mathbf{3}}_{\mathbf{F}}$ or symmetric $\mathbf{6}_{\mathbf{F}}$ representations. The spin of the light diquark is 0 for $\overline{\mathbf{3}}_{\mathbf{F}}$, while it is 1 for $\mathbf{6}_{\mathbf{F}}$. Thus, the spin of the ground state baryons is $1 / 2$ for $\overline{\mathbf{3}}_{\mathbf{F}}$, representing the $\Lambda_{b}$ and $\Xi_{b}$ baryons, while it can be both $1 / 2$ or $3 / 2$ for $\mathbf{6}_{\mathbf{F}}$, allocating $\Sigma_{b}, \Sigma_{b}^{*}, \Xi_{b}^{\prime}, \Xi_{b}^{*}, \Omega_{b}$ and $\Omega_{b}^{*}$, where the star indicates spin $3 / 2$ states. Therefore heavy hadrons form doublets. For example, $\Sigma_{b}$ and $\Sigma_{b}^{*}$ will be degenerate in the heavy quark limit, being their mass splitting caused by the chromomagnetic interaction at the order $1 / m_{Q}$. These effects can be, for example, taken into account systematically in the framework of heavy quark effective field theory. The mass difference between states belonging to the $\overline{\mathbf{3}}_{\mathbf{F}}$ and $\mathbf{6}_{\mathbf{F}}$ representations do also contain the dynamics of the light diquark subsystem, hard to accommodate in any heavy quark mass expansion. Therefore, exact solutions of the three-body problem for heavy hadrons are theoretically desirable because they will serve to test the reliability of approximate techniques: heavy quark mass expansions, variational calculations, or quark-diquark approximations.

The combined study of $Q n n$ and $Q Q n$ systems will also provide some hints to learn about the basic dynamics governing the interaction between light quarks. The interaction between pairs of quarks containing a heavy quark $Q$ is driven by the perturbative one-gluon exchange. For the $Q n n$ system the mass difference between members of the $\mathbf{6}_{\mathbf{F}}$ $\mathrm{SU}(3)$ representation comes determined only by the perturbative one-gluon exchange, while between members of the $\mathbf{6}_{\mathbf{F}}$ and $\overline{\mathbf{3}}_{\mathbf{F}}$ representations it presents contributions from the one-gluon and pseudoscalar exchanges. If the latter mass difference is attributed only to the one-gluon exchange (this would be the case of models based only on the perturbative one-gluon exchange), it will be strengthened as compared to models considering pseudoscalar potentials at the level of quarks, where a weaker one-gluon exchange will play the role. When moving to the $Q Q n$ systems only one-gluon exchange interactions between the quarks will survive, with the strength determined in the Qnn sector, where we have experimental data. This will give rise to larger masses for the ground states, due to the more attractive one-gluon exchange potential in the Qnn sector, what requires larger constituent quark masses to reproduce the experimental data.

In Ref. [15] the three-body Schrödinger equation was solved by the Faddeev method in momentum space with the constituent quark model of Ref. [16]. The results are shown in Table 2 compared to experiment and other theoretical approaches. All known experimental data are nicely described. Such an agreement and the exact method used to solve the three-body problem make our predictions also valuable as a guideline to experimentalists.

It is worth noticing that the relativistic quark-diquark approximation [19] and the harmonic oscillator variational method [18] predict a lower $3 / 2^{+}$excited state for the $\Lambda_{b}$ baryon. Such result can be easily understood by the influence of the pseudoscalar interaction between the light quarks on the $\Lambda_{b}\left(1 / 2^{+}\right)$ground state. If this attraction were 


\begin{tabular}{|cc|ccccc|}
\hline State & $J^{P}$ & {$[15]$} & {$[22]$} & {$[17]$} & {$[20]$} & {$[18]$} \\
\hline$\Xi_{b b}$ & $1 / 2^{+}$ & 10189 & 10340 & 10194 & & 10340 \\
\hline$\Delta E$ & $3 / 2^{+}$ & 29 & 30 & 41 & 20 & 27 \\
& $1 / 2^{-}$ & 217 & & 262 & & 153 \\
\hline$\Omega_{b b}$ & $1 / 2^{+}$ & 10293 & 10370 & 10267 & & 10454 \\
\hline$\Delta E$ & $3 / 2^{+}$ & 28 & 30 & 38 & 19 & 32 \\
& $1 / 2^{-}$ & 226 & & 265 & & 162 \\
\hline$\Xi_{c c}$ & $1 / 2^{+}$ & 3579 & 3660 & 3607 & 3588 & 3676 \\
\hline$\Delta E$ & $3 / 2^{+}$ & 77 & 80 & 93 & 70 & 77 \\
& $1 / 2^{-}$ & 301 & & 314 & & 234 \\
\hline$\Omega_{c c}$ & $1 / 2^{+}$ & 3697 & 3740 & 3710 & 3698 & 3815 \\
\hline$\Delta E$ & $3 / 2^{+}$ & 72 & 40 & 83 & 63 & 61 \\
& $1 / 2^{-}$ & 312 & & 317 & & 231 \\
\hline
\end{tabular}

Table 3. Ground state and excitation energies, $\Delta E$, of double charmed and bottom baryons. Masses are in MeV.

not present for the $\Lambda_{b}\left(1 / 2^{+}\right)$, the $\Lambda_{b}\left(3 / 2^{+}\right)$would be lower in mass as reported in Refs. [18, 19] (a similar effect will be observed in the charmed baryon spectra). Thus, the measurement and identification of the $\Lambda_{b}\left(3 / 2^{+}\right)$is a relevant feature that will help to clarify the nature of the interaction between the light quarks in heavy baryon spectroscopy.

Finally, we can make parameter free predictions for double charmed and bottom baryons. Our results are shown in Table 3 For double charmed baryons, the ground state is found to be at $3579 \mathrm{MeV}$, far below the result of Ref. [18] and in perfect agreement with lattice nonrelativistic QCD [20], but still a little bit higher than the non-confirmed SELEX result, $3519 \mathrm{MeV}$ [21]. It is therefore a challenge for experimentalists to confirm or to find the ground state of double charmed and bottom baryons.

\section{Summary}

To summarize this talk, we have reviewed some recent results of hadron spectroscopy and hadron-hadron interaction from the point of view of the constituent quark model. The constituent quark model is a powerful tool at our disposal to face the new issues appearing in an almost daily basis in the heavy quark sector. Unfortunately, the downsides of such robust tools lie in the risk of misusing them. Therefore, we have tried to impress upon the reader the range of validity and proper formalisms of the constituent quark model. Similarly, since there are many different models in the literature, we have tried to present conclusions that are independent of the particularities of the model chosen. We have seen how the enlargement of the Hilbert space when increasing energy, that was seen to be necessary to describe the nucleon-nucleon phenomenology above the pion production threshold, seems to be necessary to understand current problems of hadron spectroscopy. We have also tried to emphasize that spectroscopy and interaction can, and must, be understood within the same scheme when dealing with quark models. Any other alternative becomes irrelevant from the point of view of learning about properties of QCD.

It is expected that in the coming years better-quality data on all sectors discussed in this talk will become available and our results can be used to analyze these upcoming data in a model-independent way.

\section{Acknowledgments}

This work has been partially funded by the Spanish Ministerio de Educación y Ciencia and EU FEDER under Contract No. FPA2010-21750, and by the Spanish Consolider-Ingenio 2010 Program CPAN (CSD2007-00042).

\section{References}

[1] J. D. Bjorken, The November Revolution: A Theorist Reminisces, in: A Collection of Summary Talks in High Energy Physics (ed. J.D. Bjorken), p. 229 (World Scientific, New York, 2003). 
[2] W. T. Kelvin, The London, Edinburgh and Dublin Philosophical Magazine and Journal of Science, Series 6, volume 2, page 1 (1901).

[3] M. Gell-Mann, Phys. Lett. 8 (1964) 214-215.

[4] A. Valcarce, H. Garcilazo, F. Fernández, and P. González, Rep. Prog. Phys. 68 (2005) 965-1042.

[5] J. Vijande and A. Valcarce Phys. Rev. C 80 (2009) 035204(1-10).

[6] P. -O. Löwdin, J. Chem. Phys. 18 (1950) 365-376.

[7] J. Vijande, A. Valcarce, and N. Barnea, Phys. Rev. D 79 (2009) 074010(1-16).

[8] J. Vijande and A. Valcarce, Symmetry 1 (2009) 155-179.

[9] J. Vijande, E. Weissman, N. Barnea, and A. Valcarce, Phys. Rev. D 76 (2007) 094022(1-17).

[10] T. Fernández-Caramés, A. Valcarce, and J. Vijande, Phys. Rev. Lett. 103 (2009) 222001(1-4).

[11] T. F. Caramés, A. Valcarce and J. Vijande, Phys. Lett. B 699 (2011) 291-295.

[12] C. Yu-qi and W. Su-zhi, Phys. Lett. B 705 (2011) 93-97.

[13] S. Cho et al. [ExHIC Collaboration], Phys. Rev. Lett. 106 (2011) 212001(1-4).

[14] T. F. Caramés, A. Valcarce and J. Vijande, Phys. Lett. B 709 (2012) 358-361.

[15] A. Valcarce, H. Garcilazo, and J. Vijande. Eur. Phys. J. A 37 (2008) 217-225.

[16] J. Vijande, F. Fernández, and A. Valcarce, J. Phys. G 31 (2005) 481-512.

[17] B. Silvestre-Brac, Few-Body Systems 20 (1996) 1-25.

[18] W. Roberts and M. Pervin, Int. J. Mod. Phys. A23 (2008) 2817-2860.

[19] D. Ebert, R. N. Faustov, and V. O. Galkin, Phys. Lett. B 659 (2008) 612-620.

[20] N. Mathur, R. Lewis, and R. M. Woloshyn, Phys. Rev. D66 (2002) 014502(1-10).

[21] A. Ocherashvili et al. [SELEX Collaboration], Phys. Lett. B 628 (2005) 18-24.

[22] R. Roncaglia, D. B. Lichtenberg, and E. Predazzi, Phys. Rev. D 52 (1995) 1722-1725. 